\title{
Editorial Conventions
}

The main aim of the editors has been to produce a text as close to the original as possible. Within the text, spelling has been preserved as in the original, with the exception of a few expansions of otherwise unfamiliar abbreviations, such as "pd" and "ped" to "paid". Words which were inserted subsequently are silently incorporated in the text. Deletions are indicated by a note on the relevant page. Where necessary, punctuation has been inserted to clarify meaning, while obvious omissions of words have also been inserted in square brackets on the few occasions this has been needed. Contemporary abbreviations for currency have been retained with the exception of $l b$. for which we have inserted $£$. We have also normalized the writing of figures from the range of ways used by Daffy. Our best assessment of the accurate place-name is indicated in footnotes, and the same rule has been followed with other proper names.

The layout of the Account Book is by its nature the most difficult thing to translate into a printed edition, but this has been preserved so far as was practicable in order that the characteristics of the Account can be conveyed. Anthony Daffy's form of pagination was to give both the left and right hand pages of each opening of his account book the same number. As these normally formed the credit and debit pages for a single agent this was logical and we have preserved it here, distinguishing between pages by using " $A$ " for the left hand page and " $B$ " for the right hand page. One other aspect of the Account Book which is hard to reproduce is where pages or entries are crossed out. This was probably to indicate payment had been received in full. We have indicated where pages are crossed through at the end of each page entry.

Daffy made frequent and inconsistent use of horizontal lines between and in his entries, primarily, it seems, to make clear which note related to which figure. Except where these add clarity to an entry, they have been deleted. 